\title{
Application of Fullerenes in Nanomedicine: an Update
}

By: Anthony Dellinger, Zhiguo Zhou, James Connor, A.B. Madhankumar, Sarala Pamujula, Christie M. Sayes, Christopher L. Kepley

Anthony Dellinger, Zhiguo Zhou, James Connor, A.B. Madhankumar, Sarala Pamujula, Christie M. Sayes, and Christopher L. Kepley. Application of fullerenes in nanomedicine: an update. Nanomedicine, 2013 Jul; 8(7):1191-208.

Made available courtesy of Future Medicine: $\underline{\text { http://dx.doi.org/10.2217/nnm.13.99 }}$

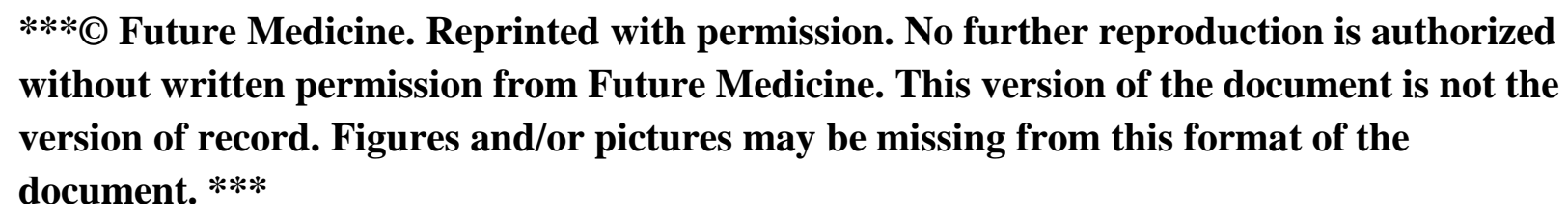

\section{Abstract:}

Fullerenes are carbon spheres presently being pursued globally for a wide range of applications in nanomedicine. These molecules have unique electronic properties that make them attractive candidates for diagnostic, therapeutic and theranostic applications. Herein, the latest research is discussed on developing fullerene-based therapeutics as antioxidants for inflammatory diseases, their potential as antiviral/bacterial agents, utility as a drug delivery device and the promise of endohedral fullerenes as new MRI contrast agents. The recent discovery that certain fullerene derivatives can stabilize immune effector cells to prevent or inhibit the release of proinflammatory mediators makes them potential candidates for several diseases such as asthma, arthritis and multiple sclerosis. Gadolinium-containing endohedral fullerenes are being pursued as diagnostic MRI contrast agents for several diseases. Finally, a new class of fullerene-based theranostics has been developed, which combine therapeutic and diagnostic capabilities to specifically detect and kill cancer cells.

Keywords: arthritis | asthma | atherosclerosis | diagnostic | fullerene | glioblastoma | multiple sclerosis | theranostic | therapeutic

\section{Article:}

\section{Fullerenes as a platform for new solutions in several medicinal areas}

Empty cage fullerenes (Figure 1A \& B) have unique electrochemical properties and have a wide range of potentially beneficial biologic properties. Another type of fullerene can have metals enclosed inside them (Figure 1C). Fullerenes have a unique cage structure with delocalized \&pi;molecular orbital electrons. This structure confers unusual activity in electron transfer systems due to their low reorganization energy, low lying excited states (singlet and triplet) and extended triplet lifetimes. Furthermore, the spherical configuration of the planar benzene rings imposes an 
unusual constraint on these \&pi;-electron orbitals. The native fullerene cage is insoluble in water and must be derivatized (simply meaning moieties or side groups must be added to the carbon cage) in order to make them water soluble (compatible in biological systems). The ability of fullerenes to be derivatized with side chains provides opportunities to diversify, manipulate and harness the electronic properties of the cage for selected applications. Of course, each derivation results in changes of the compound's physical and chemical properties, including particle size/length, \&zeta;-potential, molecular weight, surface characteristics and solubility, contributing to how they affect biological systems. All too often, results from studies examining the effects of fullerenes on biological systems tend to be extrapolated to other applications. As demonstrated below, each fullerene derivative (FD) must be assessed separately depending on the biological application. Even extremely similar FDs can have completely opposite results, which stimulates efforts to understand how changing the chemical composition and structural arrangement of fullerenes affects molecular interactions at the cellular, tissue and organ system levels. Consequently, their inherent properties described in the article, combined with their ability to be derivatized with side chains, results in almost limitless new chemical structures, making them ideal platform molecules for innovative new solutions to basic biological problems. Fullerenes are one of many types of carbon nanomaterials being investigated for next-generation medical applications; presently carbon nanotubes are being investigated as drug carriers (manipulating their hollow internal core), biosensensors (attaching enzymes to their walls) and targeted transporters of drugs ${ }^{[1-4]}$. The $2 \mathrm{D}$ structure graphene is being investigated as a drug/gene delivery nanocarrier and targeted cancer therapy ${ }^{[5,6]}$. This article will focus on the applications of both empty cage derivatized and endohedral fullerenes for therapeutic, diagnostic and theranostic (combination of diagnostic and therapeutic) applications.

\section{Fullerenes as therapeutics}

Fullerenes have been coined 'free radical sponges' and described most frequently as antioxidants, although in biological systems they can paradoxically act as both oxidants and antioxidants. The generation of free radicals such as reactive oxygen species (ROS) and reactive nitrogen species occurs naturally in cells, and their presence at sites of disease pathologies suggests they contribute to disease progression. The term free radical refers to a molecular species that possesses an unpaired electron, which makes them highly reactive. Many of the more common ROS or reactive nitrogen species that contribute to oxidative or nitrosative stress in biological systems are free radicals, including hydroxyl radicals $\left(\mathrm{OH}^{*}\right)$, superoxide anions $\left(\mathrm{O}_{2}{ }^{-}\right)$, and peroxynitrites $\left(\mathrm{ONOO}^{-}\right)$. Other ROS are not free radicals, including singlet oxygen and hydrogen peroxide $\left(\mathrm{H}_{2} \mathrm{O}_{2}\right)$, which are considered ROS because they can generate oxygen radicals, such as superoxide, via the Fenton reaction. These ROS can react with, crosslink and alter the function of many macromolecules. These species can negatively affect a wide variety of biological processes; however, in certain situations they can be beneficial to some biological processes such as signaling and cellular defenses. Antioxidants (e.g., vitamins A and C) are molecules that can eliminate or neutralize free radical electrons. This has led to a tremendous 
amount of research on preventing damage using antioxidants, theorized to, in turn, counteract disease pathologies. Indeed the perceived benefits of antioxidants are a widely accepted concept yet their use has been mainly centered on over-the-counter supplements for general health and antiaging benefits.

The carbon cage (usually $\mathrm{C}_{60}$ and $\mathrm{C}_{70}$ ) of empty cage fullerenes (Figure 1) can have antioxidant functionality based on its ability to absorb electrons and disperse them through the 3D \&pi;conjugated structure distributed over its surface. This ability to scavenge free radicals has led to their potential as a new way for treating a wide range of diseases and pathologies; these include multiple sclerosis (MS) ${ }^{[7]}$, neurodegenerative ${ }^{[8]}$, anti-HIV activity ${ }^{[9,10]}$, cancer ${ }^{[11]}$, radiation exposure $^{[12]}$, ischemia ${ }^{[13]}$, osteoporosis ${ }^{[14]}$, general inflammation ${ }^{[15]}$ and selective antimicrobial agents against bacteria ${ }^{[16]}$. Interestingly, mice and rats chronically treated with water-solubilized carboxylated fullerenes have significantly extended lifespans compared with littermate controls ${ }^{[17,18]}$. There are almost endless possibilities for adding side groups to the carbon cage to induce functionality, resulting in potential answers to some of modern medicine's most elusive problems.

\section{* Fullerenes as carriers for drug \& gene delivery}

Recently, much interest and focus has been directed towards developing safe, targeted and competent transport mechanisms for drugs and genes to cells. This transport is often riddled with difficulties as the cellular membrane creates efficient barriers that can prevent or slow the uptake of compounds. However, the small size $(<5 \mathrm{~nm})$ of fullerenes has generated significant interest in the field. Hydrophillic entities can be decorated around the fullerene cage, modifying the fullerenes relative insolubility in aqueous solutions. The water-soluble FD can readily cross the

cellular membrane without damaging cells ${ }^{[8,19]}$. These fullerenes can be further modified to become carriers of drugs and genes. Evaluated below is some of the present research in which fullerenes have been functionalized to become water soluble and utilized as versatile nanocarriers to target, treat and prevent disease with high biocompatibility.

\section{* Ability of fullerenes to affect mast cell-driven allergic inflammation}

Allergic reactions are the result of B-cell-produced, specific IgE antibody to common, normally innocuous antigens. In simplistic terms, mast cells (MCs), peripheral blood basophils (PBBs), natural killer cells, T cells and even B cells are responsible for driving the initial allergeninducing reaction through the production of IL-4 and other Th2-specific cytokines, which result in IgE sensitization. Re-exposure to the allergen triggers an allergic response through the release of inflammatory mediators from MCs and PBBs. The IgE produced binds to Fc\&epsilon;RI on MCs and PBBs, and the release of preallergic mediators is induced when two or more IgE molecules are crosslinked with the allergen. Indeed, most allergy medications are aimed at neutralizing (antihistamines and $\mathrm{H}_{1}$-receptor blockers) or preventing (omalizumab) $\mathrm{MC}$ and PBB Fc\&epsilon;RI responses. New research has demonstrated that specifically engineered FDs 
are taken up and can stabilize human tissue MCs, preventing the release of proinflammatory mediators from these cells, making them ideal candidates for those diseases controlled by MC mediators ${ }^{[20-22]}$. The ability for FDs to stabilize MC-driven diseases in vitro should translate to blocking in vivo, as shown by Dellinger et al. ${ }^{[21]}$.

\section{* Ability of fullerenes to dampen asthma pathogenesis}

MCs are ubiquitously expressed in tissues and are uniquely able to initiate and propagate certain inflammatory responses, as well as offer an interface between innate and adaptive immunity ${ }^{\text {[23] }}$. Mice without MCs fail to develop asthma-like pulmonary disease when sensitized with less aggressive immunization protocols and challenged with aerosolized allergens ${ }^{[24,25]}$. However, the strongest evidence that MCs are critical for human asthma comes from the many therapeutics used to treat the disease in humans. In general, two categories of therapies were developed to control asthma; blocking MC activation before it occurs (stabilizers) and blocking MC activation after it occurs. MCs are the only cells in the lung that have prestored TNF-[alpha] in their granules that can be immediately released upon allergen provocation ${ }^{[26]}$ and blockade of this cytokine is a valid target currently being investigated ${ }^{[27]}$. Therapies that block the effects of MCs after activation occurs are well known, commonly used, and include antihistamines and $\mathrm{H}_{1}$-receptor blockers. Thus, the importance of MCs in asthma pathogenesis is well established, and controlling the amount of mediator release from these cells is a proven drug development strategy.

The role of basophils in asthma is less clear. Their role in allergic disease has largely been viewed as redundant to that of tissue MCs. This line of thought, however, is changing with evidence that has emerged during the last 15 years. These cells are a significant source of the cytokines IL-4 and IL-13, both of which are vital to the pathogenesis of allergic disease, including asthma. It has been demonstrated that increased levels of basophils were present in the lungs of deceased asthmatics using a basophil-specific antibody developed for immunologically detecting these cell types ${ }^{[28,29]}$. Thus, the infiltration of basophils into allergic lesion sites has sparked greater interest in this once overlooked immune cell, both in adaptive as well as innate immunity ${ }^{[30]}$.

There is a strong need for novel therapeutics to treat asthmatic disease; indeed up to 55\% of patients receiving treatment for asthma have uncontrolled symptomology ${ }^{[31]}$. In some situations, especially in less severe asthmatics, asthma control is achieved with the use of nonspecific antiinflammatories, such as corticosteroids, or by the use of antihistamines and leukotriene inhibitors. However, these therapies are generally less effective in severe asthmatics or in patients with steroid resistance. As shown below, FDs are able to suppress both disease onset and reverse established disease in murine asthma models. The latter is especially important as human asthma treatment always involves established disease. 
The utility of FD's role in asthma prevention and reversal can be validated in mice treated with FDs throughout ovalbumin challenge. Treated animals have significantly less airway inflammation and bronchoconstriction compared with untreated animals. In fact, total inflammation and bronchoconstriction in the tetraglycolic acid FD-treated animals are not only significantly reduced, but are similar to that seen in nonsensitized controls (Figure 2) ${ }^{[32]}$. Thus, symptoms of disease are largely reversed in these animals. These studies used a model previously shown to utilize MCs ${ }^{[25]}$. In the established disease model, where mice were treated throughout disease development, FDs dampened eosinophilia and cytokine levels significantly in the bronchoalveolar lavage (BAL) fluid. Lung sections show massive cellular infiltration in untreated animals, while those receiving FDs have minimal cellular infiltration surrounding the airways (Figure 3). This led to reduced airway hyper-responsiveness in FD-treated animals. Thus, FDs may be useful in a clinical setting to reverse asthma pathogenesis and limit exacerbation of symptoms.

While previously published in vitro studies suggested that MC inhibition may be the predominant mechanism of FD inhibition ${ }^{[20,22]}$, in vivostudies suggest multipotent effects of these unique compounds. FD treatment reduces the levels of BAL Th2 proinflammatory cytokines and reduces lung inflammation. While IL-4 stimulates IgE production by B cells, IL-5 both recruits and activates eosinophils at the site of inflammation. FD treatment significantly reduces both IL-4 and IL-5 in the BAL fluid. Additionally, serum IgE levels were significantly reduced following FD treatment.

Importantly, the use of FD revealed a novel mechanism of action and opened up new avenues of research for asthma. Several eicosanoids (EETs) derived from the CYP450 pathway are relatively stable, and these molecules were measured in BAL fluid samples using mass spectrometry. The EETs are consistently associated with relaxation of the bronchi and other in vivo anti-inflammatory actions ${ }^{[33,34]}$. The epoxyeicosatrienoic acid was consistently upregulated in BAL fluid from FD-treated mice (Figure 4). Further in vivo studies demonstrated that the EETs play a major role in dampening the asthma phenotype as inhibitors of the EETs prevented the FD-induced modulation of the ovalbumin-induced asthma model ${ }^{[32]}$. Certain EETs stabilize human lung MCs through the inhibition of Fc\&epsilon;RI-mediator release and upregulate the CYP1B1 gene in these cells (Figure 4). Thought to be produced by lung epithelial and endothelial cells, the EETs have been shown to relax histamine-precontracted guinea pig and human bronchi ${ }^{[35,36]}$. Furthermore, they can inhibit the upregulation of VCAM-1, E-selectin, and ICAM-1, thus, potentially limiting cellular infiltration of the lung ${ }^{[37]}$. Consequently, epoxyeicosatrienoic acid upregulation plays a significant role in dampening airway inflammation and bronchoconstriction in these models. FD upregulation of EETs is a novel mechanism for controlling asthma and suggests that strategies that induce the production of EETs may be a viable therapeutic strategy for treating asthmatics.

The efficacy of FD for the treatment of asthma is illustrated through a mechanism involving the dampening of eosinophilia and cytokine levels, reducing airway hyper-responsiveness and 
upregulating EETs. Thus, FD compounds have the potential to become novel therapeutics for the treatment of asthma and pave the way for new research efforts focusing on the role of EETs in human disease.

\section{* Fullerenes inhibit inflammation caused by arthritis}

MCs play a critical role in the pathogenesis of synovitis in a murine model of rheumatoid arthritis (RA) ${ }^{[38,39]}$. The synovium of patients with RA is chronically inflamed and characterized by an expanded population of MCs, as in the mouse model. MCs are markedly increased in number and can constitute [greater than or equal] $5 \%$ of the expanded population of total synovial cells. The number of accumulated MCs differs substantially from patient to patient, in general, varying directly with the intensity of joint inflammation ${ }^{[40-42]}$. MC mediators (histamine and tryptase) are also present at higher concentrations in the synovial fluid of inflamed human joints ${ }^{[43]}$.

MC degranulation has long been associated with arthritis in several animal models, but a critical functional role in the disease was established in the K/BxN mouse model ${ }^{[39,44]}$. This arthritis model closely mimics human RA via symmetric joint involvement, chronicity, a distal-toproximal gradient of joint involvement and histological features, including synovial infiltrates, pannus, and erosions of cartilage and bone ${ }^{[45]}$. Mice deficient in MCs are highly resistant to arthritis, whereas reconstitution with normal MCs restores the wild-type phenotype. Furthermore, degranulation of MCs in the synovium is the first event observed histologically, occurring within 1-2 h of administration of $\mathrm{K} / \mathrm{BxN}$ serum ${ }^{[46]}$. Recent studies reaffirmed a role for MC mediators in arthritis ${ }^{[38]}$. Thus, MCs are a normal cell population within the human synovium and have a critical role in the pathogenesis of inflammatory arthritis.

The mitochondrial electron transport chain is the machinery that orchestrates one of the most fundamental chemical processes; the generation of cellular energy from oxygen resulting in the fuel that supports all eukaryotic life. However, it is a highly sensitive process and, when unbalanced, leads to the generation of free radicals or ROS, which have been proposed as being the mechanism underlying many chronic human diseases, including MC activation and inflammatory arthritis ${ }^{[47]}$. Fullerenes can be specifically designed to target and accumulate in the internal mitochondrial membrane bilayers. Once incorporated, FDs are positioned to neutralize superoxide molecules, reactive lipid radicals and radicals that have formed on transmembrane proteins at the site where they are generated, thus, inhibiting inflammation ${ }^{[15,21,48]}$. This, in turn, is predicted to impact diseases whose pathologies stem from radical injury.

Therefore, rationally designed FDs were synthesized to significantly block ROS production $^{[32,49]}$. While it has been shown previously that human MC degranulation in response to Fc\&epsilon;RI stimulation involves ROS ${ }^{[46]}$, it is not clear whether blocking ROS directly blocks degranulation and cytokine production. However, results suggest that blocking ROS using 
FDs in response to an immune complex (a Fc\&epsilon;RIIA-dependent stimuli ${ }^{[50]}$ ) parallels inhibition of mediator release. This is in line with previous work suggesting that FDs interfere with the generation of mitochondrial-derived ROS ${ }^{[51,52]}$.

To investigate the ability of FDs to inhibit arthritis, the K/BxN serum transfer model was used. This model induces a rapid and severe synovitis, similar to human RA, which is dependent on neutrophils, MCs and macrophages. Its MC dependence was revealed from studies in which two strains of mice deficient in this cell type, $\mathrm{W} / \mathrm{Wv}$ and $\mathrm{Sl} / \mathrm{Sld}$, were resistant to disease induction following serum transfer ${ }^{[4]}$. Susceptibility to the disease is restored in the W/Wv strain by MC engraftment. Thus, in the $\mathrm{K} / \mathrm{BxN}$ model, MCs function as a link between the serum transfer induction of autoantibodies, soluble mediators and other effector populations. By contrast, MCdeficient mice are still susceptible to collagen-induced arthritis ${ }^{[53]}$ as well as anticollagen antibody/lipopolysaccharide-induced arthritis ${ }^{[45]}$.

Previously published results have demonstrated the ability of FDs to inhibit MCmediated ${ }^{[20,22]}$ and general inflammation ${ }^{[15]}$. Given that MCs are the cellular link leading to inflammatory arthritis induction and progression, it has been hypothesized that blocking MC mediator release could block inflammatory arthritis. The liposome-encapsulated FD amphiphilic liposomal malonylfullerene (Figure 5) was administered intraperitonealy before disease induction using the $\mathrm{K} / \mathrm{BxN}$ model ${ }^{[44]}$, and during the course of disease onset there was a striking inhibition of the $\mathrm{K} / \mathrm{BxN}$-induced disease pathology. After $\mathrm{K} / \mathrm{BxN}$ serum transfer, the serumtreated mice demonstrated typical synovial hyperplasia, pannus formation and inflammatory infiltrates (Figure 6A). By contrast, amphiphilic liposomal malonylfullerene-treated animals had less evidence of clinical joint inflammation (Figure 6B) comparable to nondiseased animals (Figure 6C).

\section{* Fullerenes as a potential therapy for MS}

Experimental allergic encephalomyelitis (EAE) is a rodent model of human MS characterized by inflammation in the CNS ${ }^{[54]}$. Similar to human MS, EAE is associated with an early breach of the blood-brain barrier (BBB), focal perivascular mononuclear cell infiltrates and demyelination leading to paralysis of the extremities. While CD4 ${ }^{+} \mathrm{T}$ cells have been implicated, the underlying cause of increased vascular permeability that facilitates the entry of T cells into the CNS is unknown.

MC contribution to the pathogenesis of MS has been hypothesized based on their presence in CNS plaques of MS patients and the correlation between the number, distribution or MC markers in MS or EAE pathology ${ }^{[55]}$. Further evidence for MC involvement in EAE/MS came from studies using MC-deficient mice ${ }^{[56]}$. The MC-deficient W/Wv mice exhibited significantly reduced disease incidence, delayed disease onset and decreased mean clinical scores when compared with their wild-type congenic littermates. No differences were observed in the T- and $\mathrm{B}$-cell responses between the two groups, and reconstitution of the MC population in W/Wv 
mice restores induction of early and severe disease to wild-type levels. These data provide a new mechanism for immune destruction in EAE and indicate that MCs may be sentinels of neurologic inflammation.

Previous studies have demonstrated that fullerene-based therapeutics can significantly ameliorate $\mathrm{EAE}^{[7]}$. The FD protected neurons from oxidative and glutamate-induced injury, and restored glutamine synthesis and glutamate transporter expression in astrocytes under inflammatory insult. The in vitro efficacy translated into in vivo efficacy, as treatment initiated after disease onset reduced the clinical progression of chronic EAE in mice, suggesting this may be useful in the treatment of progressive MS and other neurodegenerative diseases.

A fullerene-based MS therapeutic was designed to specifically target axonal cells. Here a MCstabilizing FD was conjugated with tropine and an IR800 dye for tracking ${ }^{\text {[22] }}$. This MCstabilizing compound is targeted to neurons based on the affinity of tropine (a derivative of tropane containing an oxygen atom) for neuronal cell dopamine transporter receptors ${ }^{\text {[57] }}$. Thus, in addition to its ability to inhibit MC-mediator release, this compound is theorized to also target neurons and scavenge ROS. To determine if this MC-stabilizing, neuron-targeting, IR800 dye fullerene molecule could cross the BBB, mice were injected intravenously with fullerene dye, and imaged immediately and at several time points.

Initial experiments show the fullerene-dye conjugate can cross the BBB and be deposited in the brain (Figure 7). This approach using basic chemistry to add a variety of axon/neuron-targeting motifs with varying ratios of targeting motif to the fullerene core is now being used to foster maximal cell targeting and uptake. These results confirm previous studies using a $\mathrm{C}_{60}$-based formulation suggesting FDs can cross the BBB. First, mice orally treated with FDs have reduced superoxide generation in the brain ${ }^{[58]}$. Second, FDs given orally can reverse age-related changes in mitochondrial electron transport function and block age-related increases in superoxide produced by mitochondria taken from the brain ${ }^{[17,59]}$.

The SH-SY5Y human neuroblastoma cells express dopamine transporters and were differentiated into nondividing neurons using a protocol described previously ${ }^{[60]}$. A Texas $\operatorname{Red}^{\circledR}$ (Life Technologies, CA, USA)-conjugated, fullerene-tropane derivative clearly recognized the neuronal dopamine transporter receptors (Figure 8B). This is evident by the accumulation of clusters of red (from the Texas Red dye) on the membrane of the neurons and also staining of the neuronal axon. Interestingly, it appears there is an abundance of fullerene-tropane at the newly formed part of the axon (closest to the cell core) but not towards the outer axon sheath. Fullerene-tropane also penetrates into cells where there is evidence of mitochondrial colocalization (Figure 8B).

MCs are important regulators of MS ${ }^{[54-56]}$. Oxidative stress, through the generation of ROS, is an underlying mechanism that mediates MC signaling and MS pathology ${ }^{[61]}$. Indeed, several antioxidants are currently in various phases of human clinical trials (i.e., lipoic acid, inosine and 
Triomar $^{\circledR}$ [Pronova Biocare, Oslo, Norway], see ${ }^{[201]}$ ). Given that FDs can stabilize MCs ${ }^{[22]}$, are potent antioxidants ${ }^{[49,62]}$ and are anti-inflammatory medications ${ }^{[15,48]}$, FDs were tested for their ability to inhibit EAE. As seen in Figure 9, FDs reduce the clinical scores associated with MS in the EAE model, suggesting this class of rationally designed compounds may be used as a platform for new areas of therapeutic research for MS.

\section{Fullerenes as diagnostics}

Carriers for diagnostics have shown great potential in solving many present day medical imaging limitations. Endohedral fullerenes consist of a hollow core carbon shell, which during synthesis is capable of encapsulating metal ions within the cage. The endohedral fullerene cage is typically larger than the common $\mathrm{C}_{60}$. This is due to size constraints imposed by the small size of the $\mathrm{C}_{60}$ molecule; therefore, $\mathrm{C}_{80}$ molecules (or even higher: $\mathrm{C}_{82}, \mathrm{C}_{84}, \mathrm{C}_{86}, \mathrm{C}_{88}$ and $\mathrm{C}_{2 \mathrm{n}}$; i.e., $\mathrm{n}=12$, 13, 14 and so on) accommodate sufficient enclosure of desired metals, including lanthanum, yttrium, scandium and gadolinium. The endohedral fullerene's strong electronic properties can strongly enhance diagnostics. Such compounds are currently being investigated as a platform to detect cancer ${ }^{[63]}$, atherosclerosis ${ }^{[64]}$ and arthritis ${ }^{[65]}$. Present day diagnostic limitations of both sensitivity and specificity have stalled progress on accurate, enhanced patient imaging. Thus, metal endohedral fullerenes have the potential to affect the diagnosis of numerous other diagnostic pathologies.

One class of endohedral fullerene is typified by Trimetaspheres ${ }^{\circledR}$ (TMS; Luna Innovations Incorporated, VA, USA), which are $\mathrm{C}_{80}$ based with gadolinium enclosed within the cage (Figure 1C). One field in which they are being investigated for use is in MRI - a widely used diagnostic procedure that utilizes gadolinium-based contrast agents (i.e., Magnevist[trademark]; Bayer HealthCare Pharmaceuticals Inc., NJ, USA). However, several issues have limited gadolinium chelate-based contrast agents (i.e., Magnevist) for use as image-guided interventions with MRI, including gadolinium toxicity ${ }^{[66-69]}$, rapid clearance from the body, poor relaxivity-dependent sensitivity and inability to be targeted to disease-specific biomarkers. The TMSs solve many of the problems associated with current gadolinium-based contrast agents. First, the gadolinium (inside cage) that is toxic at sufficient concentrations is separated from active targeting moieties (outside cage) by an extremely stable carbon shell, possibly increasing safety. Unlike current chelates, adding targeting ligands/moieties to TMSs does not affect the ability of gadolinium to become free of the compound. Second, gadolinium-encapsulated endohedral fullerenes are more sensitive. Targeted imaging agents require strong signals by which to report the presence of an agent at a particular location. The TMS achieves 25- to 50-fold greater relaxivity compared with traditional gadolinium contrast agents. Third, the fullerene cage can be targeted to disease biomarkers, as targeting moieties can be attached to the cage (including empty cage fullerenes). Finally, the TMSs appear to be safe; no toxicity was detected in vitroand in vivo ${ }^{[49,70]}$, although further studies to assess their safety are clearly needed. 
The crux of the TMS platform for diagnosis utilizes specific biomarkers conjugated to the gadolinium-encapsulated endohedral fullerene MRI contrast agents and, in theory, could be used for any disease for which specific expression of suitable receptors exist. Two disease processes evaluated, atherosclerosis and glioblastoma, possess disease-specific biomarkers that can be targeted. Based on working prototypes, TMS endohedral fullerenes have been developed to study their cellular uptake by macrophage foam cells (for atherosclerosis) and glioblastoma cells (for cancer) in vitro . This prototype takes advantage of already identified biomarkers on diseased cells, followed by chemically attaching ligands to target the TMS to these cells (characterizing the molecules), and use of in vitro assays to determine if they bind to and enter the cells and assess any toxicological effects.

\section{* Endohedral fullerene MRI contrast agents as diagnostics for atherosclerotic plaque}

Atherosclerosis is an inflammatory disease representing a major healthcare problem in the USA. Initiation of atherosclerosis begins when blood cells (monocytes) 'stick' to blood vessel walls as a result of an individual's increased cholesterol consumption. Over time the cells continue to accumulate on vessel walls and engulf the fat droplets until large structures called plaques begin to form. The plaque can rupture and lead to complete blockage of blood flow through the vessels involved, leading to a heart attack and/or stroke. To be able to noninvasively determine the extent of plaque build up in vessel walls of at risk patients using already established diagnostic procedures would facilitate treatment options that could greatly reduce myocardial infarction and stroke incidence, which represent the most frequent causes of death in western society.

While several molecules have already been identified that are selectively expressed or highly upregulated on plaque lesions ${ }^{[71-73]}$, preliminary findings in the field focus on CD36 (macrophage scavenger receptor) as a potential target receptor. The CD36 receptor is involved in foam cell formation, mediating the influx of lipids into macrophages, possessing a promiscuous ligand binding and is highly expressed on lipid-laden macrophages of human atherosclerotic aorta $^{[74-77]}$. Different forms of oxidized phosphatidylcholine and oxidized low-density lipoprotein are high affinity ligands for CD36 ${ }^{[78]}$. The basic structure of the compounds consists of the TMS enclosed within liposomes, which was previously demonstrated ${ }^{[48]}$, with CD36 ligands decorated on the liposomes (Figure 10). As seen in Figure 11, APOE knockout mice ${ }^{[79]}$ were used to test the atherosclerotic plaque-targeting contrast agent, which serves as a working prototype for a new class of diagnostic agents that specifically attach to atherosclerotic plaques, so that previously undetected lesions can be diagnosed using MRI. The atherosclerotic plaque-targeting contrast agent clearly reveals plaque accumulation by MRI that is not seen using the same molecule without targeting ligands.

\section{Fullerenes as theranostics}

Multifunctional approaches that incorporate both medical imaging and therapy could be the future of medicine, and fullerenes prove to be an attractive platform for these approaches. This is 
due to the fullerene's ability to simultaneously detect (with biomarkers), image (with gadoliniumbased contrast) and deliver a therapeutic payload to a specified site. Progression can be monitored in real time to assess the effectiveness of treatment using direct visualization of the areas of interest through the high-contrast capabilities of endohedral fullerenes. Nanoparticles are presently being pursued for imaging and diagnostic applications ${ }^{[80,81]}$ and the next logical leap would be to couple this with patient therapy. Such multifunctional nanoplatform-based probes have shown to be effective in mouse models, including tumor detection and treatment ${ }^{[82-84]}$, as well as atherosclerosis and arthritis ${ }^{[85-87]}$.

\section{* Endohedral fullerene MRI contrast agents as theranostics for brain cancer}

Glioblastoma multiforme is an aggressive high-grade brain tumor with a poor prognosis. Ideally, a multifunctional nanoscale compound is needed that can simultaneously diagnose tumor progression and can also specifically target and kill the tumor. IL-13 receptors are selectively expressed on astrocytoma cells in gliomas. It has been previously reported that IL-13 receptortargeted chemotherapies (diphtheria toxin) ${ }^{[88,89]}$ delivered through lipid nanovesicles (liposomes) were effective in targeting and shrinking glioma tumors in a subcutaneous tumor model. MRI using gadolinium-based contrast agents has been used for diagnosing glioblastoma. IL-13 receptors are expressed on glioblastoma multiforme cells and are not significantly expressed in normal tissue ${ }^{[90]}$. In fact, many clinical trials are underway using IL-13 as a targeting moiety for various interventions of the disease ${ }^{[201]}$.

TMS was used as a platform for developing novel glioblastoma-targeting theranostics in which the diagnostic TMS is enclosed within glioblastoma-targeting IL-13 liposomes that can deliver a therapeutic payload of doxorubicin (Figure 12). After verifying in vitro binding of the glioblastoma-targeting theranostics to human glioblastoma cells, the nanoparticles were tested in vivo . As seen in Figure 13, the glioblastoma theranostic can target and shrink human brain tumors that have been transplanted in mice.

\section{Fullerene toxicity: is there concern?}

Of course, toxicity considerations are implicit when contemplating human use for novel compounds such as fullerenes. To this end there have been a number of studies examining the toxicity using a myriad of fullerene preparations. However, the results of most of these studies are conflicting, inconclusive and the subject of much debate. One such fullerene study (which was subsequently proven to be unfounded) exposed juvenile bass to nonderivatized $\mathrm{C}_{60}$, which is insoluble in water ${ }^{\text {[91] }}$. Unfortunately the authors did not include a control that provided insights into whether the observed effects were simply due to large aggregated particles, tetrahydrofuran contaminants or whether they were specific to the chemical nature of $\mathrm{C}_{60}$. However, there was widespread publicity that concluded fullerenes as a class of compounds could be toxic ${ }^{[92]}$. A subsequent publication by the same group (without publicity) demonstrated that the originally observed 'toxicity' was due to impurities in the sample ${ }^{[93]}$. Even more recently 
these original studies were formally addressed by a follow-up publication, which stated that the original Oberdorster study was 'compromised by experimental artifacts' ${ }^{[94]}$. On the opposite end of the spectrum, studies in mice demonstrate that similar $\mathrm{C}_{60}$ preparations significantly increase the lifespan of mice ${ }^{[17]}$ and rats ${ }^{[18]}$. Thus, it is difficult for researchers, and the general public, to determine if fullerenes are dangerous nanostructures that should be banned or a potential novel platform for developing new medicines. The answer to explain how a class of compounds can be simultaneously toxic and lifespan extenders will rely on well-controlled structure-activity studies.

Contributing to the confusion is that few studies examining toxicity use well characterized and highly purified material; those that are not mixtures of many isomers, aggregate sizes and impurities. Most studies in which fullerenes are deemed to be toxic use starting material with little or no characterization (e.g., dynamic light scattering, \&zeta;-potential and Fourier transform infrared spectroscopy). In addition, no studies have compared differences in cage sizes ( $\mathrm{C}_{60}$ vs $\mathrm{C}_{70}$ vs $\mathrm{C}_{80}$ ); the latter two are much more likely to become US FDA-approved products owing to their size being conducive to fewer numbers of isomers when adding side-chain moieties. The FDA requires that every new chemical entity must be evaluated separately; extrapolating toxicity (or nontoxicity) by categorizing compound mixtures and making generalizations about classes of compounds (as is the case with fullerenes) with many different isomers is not acceptable to the FDA. A fundamental understanding of basic chemistry reveals that even extremely similar molecules can have different biologic activities. There are many examples where two isomers that are very similar have completely different biological behavior. For example, the tragedy of the drug thalidomide, where one isoform was an effective sedative and the mirror image isoform was teratogenic (resulting in fetal defects), changed the face of drug testing. It is important to note that pristine nonfunctionalized fullerenes behave significantly differently to their water-soluble functionalized counterparts, furthermore each variation in fullerene functionalization should be treated and evaluated as a separate entity. This applies to the studies with fullerenes, where even extremely small changes in the core fullerene structure (through the addition of side-chain moieties) can result in the FD having completely different biological properties. Several laboratories have demonstrated this repeatedly, which highlights the difficulty in interpreting data gathered from extrapolating findings between even very similar compounds.

Complicating the task of bringing FDs to the marketplace is the fact that the FDA does not have specific guidelines for products containing nanoscale materials. A report issued by the FDA Nanotechnology Task Force (July 2007) recommends guidance by various centers within the FDA for industries working with nanomaterials ${ }^{[202]}$. Unlike 'standard' drug products, it is increasingly evident that at least in the area of characterization of nanomaterials used in drug products different standards apply. Applying small molecule principles and methodologies to nanomaterials cannot be extrapolated in biological settings. The study stressed that biodistribution analysis should be at the core of any evaluation of products containing 
nanomaterials. These biodistribution studies, as recommended by the FDA ${ }^{[202]}$, provide valuable information on where the nanoparticles are traveling and possibly accumulating, therefore, subjecting those sites to increased likelihood of toxicological effects. It was also stressed in the 2007 report that most studies (using nanomaterials) are limited in that they are short-term and might leave long-term effects unevaluated, especially because the long-term toxicity and effects for most nanoscale materials remain unknown. Furthermore, appropriate end points for in vitro assays are seen to be difficult to determine, as single cell types are often not sufficient for evaluation on the function or health of organs or tissues that are made up of multiple cell types, given that FDs are exposed to numerous types of tissues in the body. The major recommendation from the reports was that nanoscale materials be characterized with respect to size (surface area and size distribution), chemical composition (i.e., purity and crystallinity), surface structure (e.g., surface reactivity, surface groups and coatings), solubility, shape and aggregation. The protocols developed at the National Cancer Institute's, Nanotechnology Characterization Laboratories (MD, USA) was recommended as being very useful in helping to characterize nanoscale materials, and to develop standards and standardized methods for measuring nanoscale materials ${ }^{[203]}$.

At present, it is difficult to make conclusions on the absorption, distribution, metabolism and excretion profile of the many types of carbon nanomaterials presently being investigated: carbon nanotubes; nanohorns; graphene; fullerenes; and derivatives of each of these nanomaterials. This is primarily due to the relative infancy of the field and limited research on the long-term effects of fullerenes on biological systems. However, several studies have been conducted assessing the elimination of fullerenes from the body ${ }^{[19,95]}$. Researchers have concluded that there is limited absorption of fullerenes following both intravenous and oral exposure in rats. In general, elimination was rapid from the body via feces and urine ${ }^{[19,95]}$. Furthermore, several experimental studies revealed that mice treated with excessive dosages (2000 mg/kg) of fullerenes have shown no overt toxicity ${ }^{[96,97]}$. In fact, some studies have shown that fullerene treatment in mice and rats can improve cognitive function and extend lifespan by $11 \%$ (in the mouse model) and $90 \%$ (in the rat model) ${ }^{[17,18]}$. Clearly contrasting results and limited longterm data need to be addressed and efforts on creating reliable, reproducible results are still required for successful translation of these molecules into human systems. Focusing research to clarify chronic exposure to these materials, as well as identification of genotoxicity, carcinogenicity and reproductive toxicity will be paramount for encouraging fullerene studies in biological systems. Certainly, each carbon nanomaterial and specific derivatives should be carefully evaluated and characterized individually, as these materials exist in a variety of forms (e.g., carbon structure, carbon number, surface modification, charge, size, aggregation and degree of functionality). The extrapolation of results across such broad classes is not sufficient in characterizing the toxicity profiles of these molecules. Future research should be aimed at devising appropriate testing strategies to evaluate risk and toxicity profiles that do not generalize carbon nanoparticles as one, but rather evaluate each class individually. 


\section{Conclusion \& future perspective}

Fullerenes and their unique properties can be beneficial to many modern medical applications; as they are composed entirely of carbon in a hollow sphere (without requiring a toxic metal catalyst), their functionalization (with chemicals to increase their hydrophilicity) or encapsulation (in carriers, i.e., as liposomes) allows for easy solubility in biologically suitable aqueous media. The innate properties of fullerenes render high antioxidant capacity at extremely small sizes that can be functionalized or tailored to accommodate a broad range of diagnostic and therapeutic applications. Recent developments and future research in the fullerene field could reveal numerous commercial applications spanning a broad spectrum: antiviral; anticancer; photosensitizers; antioxidant activity; drug delivery and gene therapy; and highly sensitivetargeted diagnostic applications.

Current research in fullerenes could create significant strides in slowing or suppressing the progression of HIV infection to AIDS ${ }^{[98-100]}$. Mechanistically, their success as a potential antiviral application is based on the compounds unique geometry and high antioxidant activity. The compounds are capable of complexing and inhibiting the HIV protease with a relatively high structure-activity relationship ${ }^{[98]}$. The fullerenes can spatially bind in the cavity (10 $\AA$ approximate diameter) regions of HIV protease tightly and prevent the release of water, subsequently inhibiting the active regions of the protease ${ }^{[101]}$. The fullerenes strong affinity to bind to the enzymes active site results in significant inhibition of viral replication ${ }^{[99,102]}$.

Fullerenes are also capable of being photoexcited and have, thus, been exploited as photosensitizers, whereby fullerenes can be conjugated with high affinity to specific proteins and nucleic acids. The photoirradiated fullerene is excited from its ground state and converted back via intersystem crossing, creating a highly toxic singlet oxygen species. Researchers have demonstrated that using photodynamic therapy, fullerenes could photosensitize specifically targeted cells, which could rapidly reduce cell viability ${ }^{[103]}$. This technique has also been modified to a theranostic approach utilizing gadolinium-containing endohedral fullerenes to selectively target, photosensitize and image tumor reduction over time ${ }^{[104]}$.

The potential for using fullerene-based medicines is substantial but concerns of toxicity have slowed the initial enthusiasm that surrounded their discovery. Only those studies using wellcharacterized, single species, 'lead candidate' fullerene formulations can provide meaningful information regarding potential toxicological effects. Such studies are needed as the state of research today with fullerenes is shaped by studies such as these that address the observation "that extrapolation across similar nanoparticles will be dependent upon surface chemistry and concentration which may affect the degree of agglomeration and thus biological effects" ${ }^{[105]}$. Thus, more thorough studies will serve as a building block in developing a database that links surface functionalization chemistry of fullerene compounds to biological function. Significant strides have been made throughout the last decade to help propel fullerenes as a functional platform to help solve many modern medical limitations. However, the translation of successful 
research into market application will require more work to better understand uptake, biodistribution, absorption, lifetime, excretion and, ultimately, consumer safety of these nanostructures.

The unique physical and chemistry properties, the decrease in production cost, increased scalability and broad range of potential medical applications have stimulated a lot of research within the industry. While initial toxicity concerns stalled progress, recent studies have shown limited evidence of any toxicity associated with these novel nanomaterials. In time the fullerene field will make strides in penetrating industry, but only when more thorough characterization and sufficient research is accomplished to address long-term, reproducible toxicological data will this fascinating class of molecules bring advances to modern medicine.

Executive summary

\section{Fullerenes as therapeutics}

* Specifically engineered fullerene derivatives (FDs) are taken up and can stabilize human mast cells by preventing the release of proinflammatory mediators.

* FDs have the potential to become novel therapeutics for the treatment of asthma, illustrated through a mechanism involving the dampening of eosinophilia and cytokine levels, reducing airway hyper-responsiveness and upregulating eicosanoids.

* FD-treated mice demonstrated a significant reduction in both the clinical indices as well as ankle swelling measurements.

* FDs reduce the clinical scores associated with multiple sclerosis in the experimental allergic encephalomyelitis model.

* Rationally designed fullerene compounds may be used as a platform for new areas of therapeutic research for those diseases influenced by mast cell mediators.

\section{Fullerenes as diagnostics}

* Gadolinium-containing fullerenes can serve as a platform for developing future biomarkertargeting contrast agents.

* Gadolinium endohedral fullerene-based MRI contrast agents can be functionalized to specifically target atherosclerotic plaques.

\section{Fullerenes as theranostics}

* Specifically engineered gadolinium endohedral fullerene MRI contrast agents can cross the blood-brain barrier to selectively target and deliver a therapeutic payload to cancerous glioblastoma cells. 


\section{Fullerene toxicity: is there concern?}

* Studies on the toxicity of FDs have produced conflicting results in the scientific community; often experimental results are extrapolated across a diverse family of carbon-based nanomaterials.

* Future research should be aimed at devising appropriate testing strategies to evaluate risk and toxicity profiles that do not generalize nanoparticles as one class, but rather evaluate each class individually.

\section{CAPTION(S):}

Figure 1. Representative fullerene structures.

(A) Empty cage $\mathrm{C}_{60}$ fullerene, (B) empty cage $\mathrm{C}_{70}$ fullerene and (C) gadolinium-enclosed $\mathrm{C}_{80}$ endohedral fullerene.

\section{References}

Papers of special note have been highlighted as: * of interest $* *$ of considerable interest

1 Rao CNR, Cheetham AK. Science and technology of nanomaterials: current status and future prospects. J. Mater. Chem. 11(12), 2887-2894 (2001).

2 Liu Z, Robinson JT, Tabakman SM, Yang K, Dai HJ. Carbon materials for drug delivery and cancer therapy. Mater. Today 14(7-8), 316-323 (2011).

3 Lu FS, Gu LR, Meziani MJ et al. Advances in bioapplications of carbon nanotubes. Adv. Mater. 21(2), 139-152 (2009).

4 Sun Z, Liu Z, Meng J et al. Carbon nanotubes enhance cytotoxicity mediated by human lymphocytes in vitro . PloS One 6(6), e21073 (2011).

5 Shen H, Zhang LM, Liu M, Zhang ZJ. Biomedical applications of graphene. Theranostics 2(3), 283-294 (2012).

6 Feng LZ, Zhang SA, Liu ZA. Graphene based gene transfection. Nanoscale 3(3), 1252-1257 (2011).

7 Basso AS, Frenkel D, Quintana FJ et al. Reversal of axonal loss and disability in a mouse model of progressive multiple sclerosis. J. Clin. Invest. 118(4), 1532-1543 (2008).

8 Dugan LL, Turetsky DM, Du C et al. Carboxyfullerenes as neuroprotective agents. Proc. Natl Acad. Sci. USA 94(17), 9434-9439 (1997). 
9 Bosi S, Da RT, Spalluto G, Balzarini J, Prato M. Synthesis and anti-HIV properties of new water-soluble bis-functionalized[60]fullerene derivatives. Bioorg. Med. Chem. Lett. 13(24), 4437-4440 (2003).

10 Marchesan S, Da RT, Spalluto G, Balzarini J, Prato M. Anti-HIV properties of cationic fullerene derivatives. Bioorg. Med. Chem. Lett. 15(15), 3615-3618 (2005).

11 Berger CS, Marks JW, Bolskar RD, Rosenblum MG, Wilson LJ. Cell internalization studies of gadofullerene-(ZME-018) immunoconjugates into A375m melanoma cells. Transl. Oncol. 4(6), 350-354 (2011).

12 Daroczi B, Kari G, McAleer MF, Wolf JC, Rodeck U, Dicker AP. In vivo radioprotection by the fullerene nanoparticle DF-1 as assessed in a zebrafish model. Clin. Cancer Res. 12(23), 7086-7091 (2006).

13 Lai YL, Murugan P, Hwang KC. Fullerene derivative attenuates ischemia-reperfusioninduced lung injury. Life Sci. 72(11), 1271-1278 (2003).

14 Gonzalez KA, Wilson LJ, Wu W, Nancollas GH. Synthesis and in vitro characterization of a tissue-selective fullerene: vectoring $\mathrm{C}(60)(\mathrm{OH})(16) \mathrm{AMBP}$ to mineralized bone. Bioorg. Med. Chem. 10(6), 1991-1997 (2002).

15 Dellinger A, Zhou Z, Lenk R, Macfarland D, Kepley CL. Fullerene nanomaterials inhibit phorbol myristate acetate-induced inflammation. Exp. Dermatol. 18(12), 1079-1081 (2009).

16 Tsao N, Luh TY, Chou CK et al. In vitro action of carboxyfullerene. J. Antimicrob. Chemother. 49(4), 641-649 (2002).

17 Quick KL, Ali SS, Arch R, Xiong C, Wozniak D, Dugan LL. A carboxyfullerene SOD mimetic improves cognition and extends the lifespan of mice. Neurobiol. Aging 29(1), 117-128 (2008).

** Fullerenes used as the first demonstration of administered antioxidants with mitochondrial activity and nervous system penetration not only increased lifespan, but also rescued age-related cognitive impairment in mammals.

18 Baati T, Bourasset F, Gharbi $\mathrm{N}$ et al. The prolongation of the lifespan of rats by repeated oral administration of [60]fullerene. Biomaterials33(19), 4936-4946 (2012).

* Revealed that aqueous fullerene suspensions not only failed to produce acute or subacute toxicity in rodents, but could also protect their livers in a dose-dependent manner against free radical damage and prolong the lifespan of rats by nearly $100 \%$ without showing toxic effects. 
19 Yamago S, Tokuyama H, Nakamura E et al. In vivo biological behavior of a water-miscible fullerene: ${ }^{14} \mathrm{C}$ labeling, absorption, distribution, excretion and acute toxicity. Chem. Biol. 2(6), 385-389 (1995).

20 Ryan JJ, Bateman HR, Stover A et al. Fullerene nanomaterials inhibit the allergic response. J. Immunol. 179(1), 665-672 (2007).

** Identifies a new biological function for fullerenes and may represent a novel way to control mast cell-dependent diseases, including asthma, inflammatory arthritis, heart disease and multiple sclerosis.

21 Dellinger A, Zhou Z, Norton SK, Lenk R, Conrad D, Kepley CL. Uptake and distribution of fullerenes in human mast cells. Nanomedicine6(4), 575-582 (2010).

22 Norton SK, Dellinger A, Zhou Z et al. A new class of human mast cell and peripheral blood basophil stabilizers that differentially control allergic mediator release. Clin. Transl. Sci. 3(4), 158-169 (2010).

23 Galli SJ, Tsai M, Piliponsky AM. The development of allergic inflammation. Nature 454(7203), 445-454 (2008).

24 Kobayashi T, Miura T, Haba T et al. An essential role of mast cells in the development of airway hyperresponsiveness in a murine asthma model. J. Immunol. 164(7), 3855-3861 (2000).

25 Williams CM, Galli SJ. Mast cells can amplify airway reactivity and features of chronic inflammation in an asthma model in mice. J. Exp. Med.192(3), 455-462 (2000).

26 Gordon JR, Galli SJ. Mast cells as a source of both preformed and immunologically inducible TNF-alpha/cachectin. Nature 346, 274-276 (1990).

27 Brightling C, Berry M, Amrani Y. Targeting TNF-alpha: a novel therapeutic approach for asthma. J. Allergy Clin. Immunol. 121(1), 5-10 (2008).

28 Kepley CL, Mcfeeley PJ, Oliver JM, Lipscomb MF. Immunohistochemical detection of human basophils in postmortem cases of fatal asthma.Am. J. Respir. Crit. Care Med. 164(6), 1053-1058 (2001).

29 Kepley CL, Craig SS, Schwartz LB. Identification and partial characterization of a unique marker for human basophils. J. Immunol.154(12), 6548-6555 (1995).

30 Schroeder JT. Basophils beyond effector cells of allergic inflammation. Adv. Immunol. 101, 123-161 (2009).

31 Jackson DJ, Sykes A, Mallia P, Johnston SL. Asthma exacerbations: origin, effect, and prevention. J. Allergy Clin. Immunol. 128(6), 1165-1174 (2011). 
32 Norton SK, Wijesinghe DS, Dellinger A et al. Epoxyeicosatrienoic acids are involved in the C(70) fullerene derivative-induced control of allergic asthma. J. Allergy Clin. Immunol. 130(3), 761-769 (2012).

** Fullerene-treated mice were found to have significantly reduced airway inflammation, eosinophilia and bronchoconstriction associated with allergic asthma.

33 Sudhahar V, Shaw S, Imig JD. Epoxyeicosatrienoic acid analogs and vascular function. Curr. Med. Chem. 17(12), 1181-1190 (2010).

34 Pfister SL, Gauthier KM, Campbell WB. Vascular pharmacology of epoxyeicosatrienoic acids. Adv. Pharmacol. 60, 27-59 (2010).

35 Morin C, Rousseau E. Effects of 5-oxo-ETE and 14,15-EET on reactivity and $\mathrm{Ca}^{2+}$ sensitivity in guinea pig bronchi. Prostaglandins Other Lipid Mediat. 82(1-4), 30-41 (2007).

36 Morin C, Sirois M, Echave V, Gomes MM, Rousseau E. EET displays anti-inflammatory effects in TNF-alpha stimulated human bronchi: putative role of CPI-17. Am. J. Respir. Cell Mol. Biol. 38(2), 192-201 (2008).

37 Node K, Huo Y, Ruan X et al. Anti-inflammatory properties of cytochrome P450 epoxygenase-derived eicosanoids. Science 285(5431), 1276-1279 (1999).

38 Nigrovic PA, Lee DM. Synovial mast cells: role in acute and chronic arthritis. Immunol. Rev. 217, 19-37 (2007).

39 Woolley DE. The mast cell in inflammatory arthritis. N. Engl. J. Med. 348(17), 1709-1711 (2003).

40 Irani AA, Golzar N, Deblois G, Gruber B, Schwartz LB. Distribution of mast cell subsets in rheumatoid arthritis and osteoarthritis synovia.Arthritis Rheum. 30, 66 (1987).

41 Bridges AJ, Malone DG, Jicinsky J et al. Human synovial mast cell involvement in rheumatoid arthritis and osteoarthritis: relationship to disease type, clinical activity, and antirheumatic therapy. Arthritis Rheum. 34, 1116-1124 (1991).

42 Gotis-Graham I, Smith MD, Parker A, Mcneil HP. Synovial mast cell responses during clinical improvement in early rheumatoid arthritis.Ann. Rheum. Dis. 57(11), 664-671 (1998).

43 Lavery JP, Lisse JR. Preliminary study of the tryptase levels in the synovial fluid of patients with inflammatory arthritis. Ann. Allergy 72, 425-427 (1994).

44 Lee DM, Friend DS, Gurish MF, Benoist C, Mathis D, Brenner MB. Mast cells: a cellular link between autoantibodies and inflammatory arthritis. Science. 297(5587), 1689-1692 (2002). 
45 Dinser R. Animal models for arthritis. Best Pract. Res. Clin. Rheumatol. 22(2), 253-267 (2008).

46 Nigrovic PA, Lee DM. Mast cells in autoantibody responses and arthritis. Novartis Found. Symp. 271, 200-209 (2005).

47 Winyard PG, Ryan B, Eggleton P et al. Measurement and meaning of markers of reactive species of oxygen, nitrogen and sulfur in healthy human subjects and patients with inflammatory joint disease. Biochem. Soc. Trans. 39, 1226-1232 (2011).

48 Zhou Z, Lenk RP, Dellinger A, Wilson SR, Sadler R, Kepley CL. Liposomal formulation of amphiphilic fullerene antioxidants. Bioconjug. Chem. 21(9), 1656-1661 (2010).

49 Ehrich M, van Tassell R, Li Y, Zhou Z, Kepley CL. Fullerene antioxidants decrease organophosphate-induced acetylcholinesterase inhibitionin vitro . Toxicol. In Vitro 25(1), 301307 (2011).

50 Zhao W, Gomez G, Macey M, Kepley CL, Schwartz LB. In vitro desensitization of human skin mast cells. J. Clin. Immunol. 32(1), 150-160 (2011).

51 Chirico F, Fumelli C, Marconi A et al. Carboxyfullerenes localize within mitochondria and prevent the UVB-induced intrinsic apoptotic pathway. Exp. Dermatol. 16(5), 429-436 (2007).

52 Fumelli C, Marconi A, Salvioli S et al. Carboxyfullerenes protect human keratinocytes from ultraviolet-B-induced apoptosis. J. Invest. Dermatol. 115(5), 835-841 (2000).

53 Pitman N, Asquith DL, Murphy G, Liew FY, Mcinnes IB. Collagen-induced arthritis is not impaired in mast cell-deficient mice. Ann. Rheum. Dis. 70(6), 1170-1171 (2011).

54 Brown MA, Tanzola MB, Robbie-Ryan M. Mechanisms underlying mast cell influence on EAE disease course. Mol. Immunol. 38(16-18), 1373-1378 (2002).

55 Gregory GD, Bickford A, Robbie-Ryan M, Tanzola M, Brown MA. MASTering the immune response: mast cells in autoimmunity. Novartis Found. Symp. 271, 215-225; discussion 225-231 (2005).

56 Secor VH, Secor WE, Gutekunst CA, Brown MA. Mast cells are essential for early onset and severe disease in a murine model of multiple sclerosis. J. Exp. Med. 191(5), 813-822 (2000).

$57 \mathrm{Li} \mathrm{LB}$, Reith ME. Modeling of the interaction of $\mathrm{Na}^{+}$and $\mathrm{K}^{+}$with the binding of dopamine and $\left[{ }^{3}\right.$ H]WIN 35,428 to the human dopamine transporter. J. Neurochem. 72(3), 1095-1109 (1999). 
58 Dugan LL, Gabrielsen JK, Yu SP, Lin TS, Choi DW. Buckminsterfullerenol free radical scavengers reduce excitotoxic and apoptotic death of cultured cortical neurons. Neurobiol. Dis. 3(2), 129-135 (1996).

59 Ali SS, Hardt JI, Quick KL et al. A biologically effective fullerene $\left(\mathrm{C}_{60}\right)$ derivative with superoxide dismutase mimetic properties. Free Radic. Biol. Med. 37(8), 1191-1202 (2004).

60 Borland MK, Trimmer PA, Rubinstein JD et al. Chronic, low-dose rotenone reproduces Lewy neurites found in early stages of Parkinson's disease, reduces mitochondrial movement and slowly kills differentiated SH-SY5Y neural cells. Mol. Neurodegener. 3, 21 (2008).

61 Gilgun-Sherki Y, Melamed E, Offen D. The role of oxidative stress in the pathogenesis of multiple sclerosis: the need for effective antioxidant therapy. J. Neurol. 251(3), 261-268 (2004).

62 Bakry R, Vallant RM, Najam-Ul-Haq M et al. Medicinal applications of fullerenes. Int. J. Nanomedicine 2(4), 639-649 (2007).

63 Mody VV, Nounou MI, Bikram M. Novel nanomedicine-based MRI contrast agents for gynecological malignancies. Adv. Drug Deliv. Rev.61(10), 795-807 (2009).

64 Nitta N, Seko A, Sonoda A et al. Is the use of fullerene in photodynamic therapy effective for atherosclerosis? Cardiovasc. Intervent. Radiol.31(2), 359-366 (2008).

65 Bolskar RD. Gadofullerene MRI contrast agents. Nanomedicine (Lond.) 3(2), 201-213 (2008).

66 Chewning RH, Murphy KJ. Gadolinium-based contrast media and the development of nephrogenic systemic fibrosis in patients with renal insufficiency. J. Vasc. Interv. Radiol. 18(3), 331-333 (2007).

67 Runge VM. Gadolinium and nephrogenic systemic fibrosis. AJR Am. J. Roentgenol. 192(4), W195-W196 (2009).

68 Ledneva E, Karie S, Launay-Vacher V, Janus N, Deray G. Renal safety of gadolinium-based contrast media in patients with chronic renal insufficiency. Radiology 250(3), 618-628 (2009).

69 Cowper SE. Nephrogenic systemic fibrosis: a review and exploration of the role of gadolinium. Adv. Dermatol. 23, 131-154 (2007).

70 Macfarland DK, Walker KL, Lenk RP et al. Hydrochalarones: a novel endohedral metallofullerene platform for enhancing magnetic resonance imaging contrast. J. Med. Chem. 51(13), 3681-3683 (2008).

71 Ouimet T, Lancelot E, Hyafil F et al. Molecular and cellular targets of the MRI contrast agent p947 for atherosclerosis imaging. Mol. Pharm.9(4), 850-861 (2012). 
72 Uno K, Nicholls SJ. Biomarkers of inflammation and oxidative stress in atherosclerosis. Biomark. Med. 4(3), 361-373 (2010).

73 Sadeghi MM, Glover DK, Lanza GM, Fayad ZA, Johnson LL. Imaging atherosclerosis and vulnerable plaque. J. Nucl. Med. 51(Suppl. 1), 51S-65S (2010).

74 Nergiz-Unal R, Rademakers T, Cosemans JM, Heemskerk JW. CD36 as a multiple-ligand signaling receptor in atherothrombosis. Cardiovasc. Hematol. Agents Med. Chem. 9(1), 42-55 (2011).

75 Collot-Teixeira S, Martin J, Rmott-Roe C, Poston R, Mcgregor JL. CD36 and macrophages in atherosclerosis. Cardiovasc. Res. 75(3), 468-477 (2007).

76 Ge Y, Elghetany MT. CD36: a multiligand molecule. Lab. Hematol. 11(1), 31-37 (2005).

77 Silverstein RL, Febbraio M. CD36, a scavenger receptor involved in immunity, metabolism, angiogenesis, and behavior. Sci. Signal. 2(72), re3 (2009).

78 Podrez EA, Poliakov E, Shen Z et al. Identification of a novel family of oxidized phospholipids that serve as ligands for the macrophage scavenger receptor CD36. J. Biol. Chem. 277(41), 38503-38516 (2002).

79 Kolovou G, Anagnostopoulou K, Mikhailidis DP, Cokkinos DV. Apolipoprotein E knockout models. Curr. Pharm. Des. 14(4), 338-351 (2008).

80 Patra CR, Jing Y, Xu YH et al. A core-shell nanomaterial with endogenous therapeutic and diagnostic functions. Cancer Nanotechnol. 1(1), 13-18 (2010).

81 Yigit MV, Zhu L, Ifediba MA et al. Noninvasive MRI-SERS imaging in living mice using an innately bimodal nanomaterial. ACS Nano 5(2), 1056-1066 (2011).

82 Park JH, von Maltzahn G, Ruoslahti E, Bhatia SN, Sailor MJ. Micellar hybrid nanoparticles for simultaneous magnetofluorescent imaging and drug delivery. Angew. Chem. Int. Ed. Engl. 47(38), 7284-7288 (2008).

83 Park JH, Von Maltzahn G, Zhang L et al. Magnetic iron oxide nanoworms for tumor targeting and imaging. Adv. Mater. 20(9), 1630-1635 (2008).

84 Von Maltzahn G, Ren Y, Park JH et al. In vivo tumor cell targeting with "click" nanoparticles. Bioconjug. Chem. 19(8), 1570-1578 (2008).

85 Jaffer FA, Libby P, Weissleder R. Molecular and cellular imaging of atherosclerosis: emerging applications. J. Am. Coll. Cardiol. 47(7), 1328-1338 (2006). 
86 Jaffer FA, Nahrendorf M, Sosnovik D, Kelly KA, Aikawa E, Weissleder R. Cellular imaging of inflammation in atherosclerosis using magnetofluorescent nanomaterials. Mol. Imaging 5(2), 85-92 (2006).

87 Mccarthy JR, Jaffer FA, Weissleder R. A macrophage-targeted theranostic nanoparticle for biomedical applications. Small 2(8-9), 983-987 (2006).

88 Li C, Hall WA, Jin N, Todhunter DA, Panoskaltsis-Mortari A, Vallera DA. Targeting glioblastoma multiforme with an IL-13/diphtheria toxin fusion protein in vitro and in vivo in nude mice. Protein Eng. 15(5), 419-427 (2002).

89 Todhunter DA, Hall WA, Rustamzadeh E, Shu Y, Doumbia SO, Vallera DA. A bispecific immunotoxin (DTAT13) targeting human IL-13 receptor (IL-13R) and urokinase-type plasminogen activator receptor (uPAR) in a mouse xenograft model. Protein Eng. Des. Sel. 17(2), 157-164 (2004).

90 Madhankumar AB, Slagle-Webb B, Mintz A, Sheehan JM, Connor JR. Interleukin13 receptor-targeted nanovesicles are a potential therapy for glioblastoma multiforme. Mol. Cancer Ther. 5(12), 3162-3169 (2006).

91 Oberdorster E. Manufactured nanomaterials (fullerenes, $\mathrm{C}_{60}$ ) induce oxidative stress in the brain of juvenile largemouth bass. Environ. Health Perspect. 112(10), 1058-1062 (2004).

92 Barnaby JF. Study raises concerns about carbon particles. New York Times , 29th March (2004).

93 Zhu S, Oberdorster E, Haasch ML. Toxicity of an engineered nanoparticle (fullerene, $\mathrm{C}_{60}$ ) in two aquatic species, Daphnia and fathead minnow. Mar. Environ. Res. 62(Suppl.), S5-S9 (2006).

94 Henry TB, Petersen EJ, Compton RN. Aqueous fullerene aggregates ( $\mathrm{nC}_{60}$ ) generate minimal reactive oxygen species and are of low toxicity in fish: a revision of previous reports. Curr. Opin. Biotechnol. 22(4), 533-537 (2011).

95 Chen HH, Yu C, Ueng TH et al. Acute and subacute toxicity study of water-soluble polyalkylsulfonated C 60 in rats. Toxicol. Pathol. 26(1), 143-151 (1998).

96 Mori T, Takada H, Ito S, Matsubayashi K, Miwa N, Sawaguchi T. Preclinical studies on safety of fullerene upon acute oral administration and evaluation for no mutagenesis. Toxicology 225(1), 48-54 (2006).

97 Gharbi N, Pressac M, Hadchouel M, Szwarc H, Wilson SR, Moussa F. [60]fullerene is a powerful antioxidant in vivo with no acute or subacute toxicity. Nano. Lett. 5(12), 2578-2585 (2005). 
98 Ibrahim M, Saleh NA, Elshemey WM, Elsayed AA. Fullerene derivative as anti-HIV protease inhibitor: molecular modeling and QSAR approaches. Mini. Rev. Med. Chem. 12(6), 447-451 (2012).

99 Luo Z, Xu X, Zhang X, Hu L. Development of calixarenes, cyclodextrins and fullerenes as new platforms for anti-HIV drug design: an overview. Mini Rev. Med. Chem. 13(8), 1160-1165 (2013).

100 Kornev AB, Peregudov AS, Martynenko VM, Balzarini J, Hoorelbeke B, Troshin PA. Synthesis and antiviral activity of highly water-soluble polycarboxylic derivatives of [70]fullerene. Chem. Commun. (Camb.) 47(29), 8298-8300 (2011).

101 Zhu ZW, Schuster DI, Tuckerman ME. Molecular dynamics study of the connection between flap closing and binding of fullerene-based inhibitors of the HIV-1 protease. Biochemistry 42(5), 1326-1333 (2003).

102 Marcorin GL, Da Ros T, Castellano S et al. Design and synthesis of novel [60]fullerene derivatives as potential HIV aspartic protease inhibitors. Org. Lett. 2(25), 3955-3958 (2000).

103 Rancan F, Rosan S, Boehm F et al. Cytotoxicity and photocytotoxicity of a dendritic C-60 mono-adduct and a malonic acid C-60 tris-adduct on Jurkat cells. J. Photoch. Photobio. B 67(3), 157-162 (2002).

104 Liu Y, Wang H. Nanomedicine: nanotechnology tackles tumours. Nat. Nanotechnol. 2(1), 20-21 (2007).

105 Saathoff JG, Inman AO, Xia XR, Riviere JE, Monteiro-Riviere NA. In vitro toxicity assessment of three hydroxylated fullerenes in human skin cells. Toxicol. In Vitro 25(8), 21052112 (2011).

\section{* Websites}

201 ClinicalTrials.gov.http://clinicaltrials.gov/

202 US FDA.

Nanotechnology.http://www.fda.gov/ScienceResearch/SpecialTopics/Nanotechnology/

203 National Cancer Institute. Nanotechnology Characterization Laboratory

Objectives.http://ncl.cancer.gov/objectives_ncl_obj1.asp

\section{Author Affiliation(s):}

${ }^{[1]}$ Joint School of Nanoscience \& Nanoengineering, 2907 East Lee Street, Greensboro, NC 27401, USA 
${ }^{[2]}$ Luna Innovations Incorporated, nanoWorks Division, 521 Bridge Street, Danville, VA 24541, USA

${ }^{[3]}$ Penn State University, Department of Neurosurgery, Hershey Medical Center, 500 University Drive, Hershey, PA 17033, USA

${ }^{[4]}$ Center for Aerosol \& Nanomaterials Engineering, RTI International, Research Triangle Park, NC 27709, USA

${ }^{[1]}$ Joint School of Nanoscience \& Nanoengineering, 2907 East Lee Street, Greensboro, NC 27401, USA. clkepley@uncg.edu

\section{Author Note(s):}

* Author for correspondence

\section{Financial \& competing interests disclosure}

CL Kepley has received the following grants: 1R01GM083274-01C and R21 ESO15696-01A1. The authors have no other relevant affiliations or financial involvement with any organization or entity with a financial interest in or financial conflict with the subject matter or materials discussed in the manuscript apart from those disclosed.

No writing assistance was utilized in the production of this manuscript. 